\title{
AN EMBEDDING THEOREM FOR FREE INVERSE SEMIGROUPS
}

\author{
by W. D. MUNN
}

(Received 14 February, 1980)

In this note it is shown that if $S$ is a free inverse semigroup of rank at least two and if $e, f$ are idempotents of $S$ such that $e>f$ then $S$ can be embedded in the partial semigroup $e S e \backslash f S f$. The proof makes use of Scheiblich's construction for free inverse semigroups $[7,8]$ and of Reilly's characterisation of a set of free generators in an inverse semigroup $[4,5]$.

The terminology and notation throughout is that of Howie [2]. For any two sets $M$ and $N$ we denote by $M \backslash N$ the set of all elements of $M$ that are not in $N$.

Let $X$ be a nonempty set and let $G$ denote the free group $\mathscr{F} \mathscr{G}_{X}$ on $X[1, \mathrm{Ch}$. 7]. The elements of $G$ are the 'reduced words' in the alphabet $X \cup X^{-1}$, where $X^{-1}$ denotes the set of formal inverses of the elements of $X$, and the identity of $G$ is the empty word 1 . The length (more precisely, the $X$-length) $l(a)$ of $a \in G$ is defined by

$$
l(a)= \begin{cases}n & \text { if the reduced form of } a \text { is } x_{1} x_{2} \ldots x_{n}\left(x_{i} \in X \cup X^{-1}\right), \\ 0 & \text { if } a=1\end{cases}
$$

The cardinal number $|X|$ of $X$ is called the rank of $G$. By [1, Theorem 7.3.3], two free groups are isomorphic if and only if they have the same rank.

For all $a \in G$ the set of all initial segments of $a$ (including 1 ) will be denoted by $\bar{a}$ and for all nonempty subsets $A$ of $G$ we write $\bar{A}=\{\bar{a}: a \in A\}$. We say that $A$ is left closed if and only if $A=\bar{A}$.

Scheiblich $[7,8]$ constructs the free inverse semigroup on $X$ as follows. Let $Y$ denote the set of all finite left closed subsets of $G$ having at least two elements and let

$$
S=\{(A, g) \in \mathcal{Y} \times G: g \in A\} .
$$

If $(A, g),(B, h) \in S$ then $A \cup g B \in \mathscr{Y}$, as is easily checked: consequently, a multiplication can be defined on $S$ by the rule that

$$
(A, g)(B, h)=(A \cup g B, g h) \text {. }
$$

With respect to this multiplication $S$ is an inverse semigroup in which

$$
(\forall(A, g) \in S) \quad(A ; g)^{-1}=\left(g^{-1} A, g^{-1}\right) .
$$

Let $\kappa: X \rightarrow S$ denote the mapping $x \mapsto(\bar{x}, x)$. Then to each mapping $\theta$ from $X$ into an arbitrary inverse semigroup $T$ there corresponds a unique homomorphism $\phi: S \rightarrow T$ such that $\theta=\kappa \phi$. Thus $S$ is the free inverse semigroup on $X$. It will be denoted here by $\mathscr{F}_{X}$.

We call $|X|$ the rank of $S$. It can be shown that two free inverse semigroups are isomorphic if and only if they have the same rank (see [6, Corollary 1.5]).

Glasgow Math. J. 22 (1981) 217-222. 
The semilattice of $S$ is the set

$$
E(S)=\{(A, 1): A \in \mathcal{Y}\}
$$

and its partial ordering is given by the rule:

$$
(\forall A, B \in \mathcal{Y}) \quad(A, 1) \geq(B, 1) \Leftrightarrow A \subseteq B .
$$

Let $(A, 1),(B, 1) \in E(S)$. We say that $(A, 1)$ covers $(B, 1)$ if and only if $(A, 1)>(B, 1)$ and there is no $(C, 1) \in E(S)$ such that $(A, 1)>(C, 1)>(B, 1)$. Thus $(A, 1)$ covers $(B, 1)$ if and only if $A \subset B$ and $|A|+1=|B|$.

The mapping $\pi: S \rightarrow G$ defined by

$$
(A, g) \pi=g
$$

is evidently a surjective homomorphism (and $\pi^{\circ} \pi^{-1}$ is the least group congruence on $S$ [2, Ch. V, §3]). Let us denote the $\mathscr{Y}$-component of a typical element $a$ of $S$ by $\mathscr{S}(a)$. This provides us with the following notation for the elements of $S$ :

$$
(\forall a \in S) \quad a=(\mathscr{P}(a), a \pi) .
$$

Let $K$ be a nonempty subset of $S$. Then the inverse subsemigroup of $S$ generated by $K$ is the subsemigroup $\left\langle K \cup K^{-1}\right\rangle$ generated by $K \cup K^{-1}$, where $K^{-1}$ denotes $\left\{k^{-1}: k \in K\right\}$. We say that $K$ is a set of free generators of $\left\langle K \cup K^{-1}\right\rangle$ if and only if to each mapping $\theta$ from $K$ into an arbitrary inverse semigroup $T$ there corresponds a unique homomorphism $\phi:\left\langle K \cup K^{-1}\right\rangle \rightarrow T$ such that $\theta=\iota \phi$, where $\iota$ denotes the inclusion mapping $K \rightarrow$ $\left\langle K \cup K^{-1}\right\rangle[4,5]$. If $K$ is a set of free generators of $\left\langle K \cup K^{-1}\right\rangle$ then $\left\langle K \cup K^{-1}\right\rangle$ is a free inverse subsemigroup of $S$ of rank $|K|$. Note, in particular, that $\{(\bar{x}, x): x \in X\}$ is a set of free generators of $S$.

Reilly has provided a useful criterion for a nonempty subset $K$ of $S$ to be a set of free generators of $\left\langle K \cup K^{-1}\right\rangle[4$, Theorem 2.2 and remark on p. 417; 5]. We state his result as

LEMMA 1. Let $S=\mathscr{F} \Phi_{X}$ and let $K$ be a nonempty subset of $S$. Then $K$ is a set of free generators of $\left\langle K \cup K^{-1}\right\rangle$ if and only if it satisfies the following two conditions:

(i) $K \cap K^{-1}=\varnothing$,

(ii) if $v \in K \cup K^{-1}$ is such that

$$
\mathscr{S}(v) \subseteq \bigcup_{i=1}^{n} \mathscr{S}\left(v_{i, 1} v_{i, 2} \ldots v_{i, n_{i}}\right)
$$

where each $v_{i, j} \in K \cup K^{-1}$ and $v_{i, j}^{-1} \neq v_{i, j+1}\left(j=1,2, \ldots, n_{i}-1 ; i=1,2, \ldots, n\right)$, then $v=v_{i, 1}$ for some $i$.

Our main theorem follows below.

THEOREM 1. Let $S=F \mathscr{I}_{X}$, where $|X| \geq 2$, and let $e, f \in E(S)$ be such that $e>f$. Then $S$ can be embedded in the partial semigroup eSe $\backslash f S f$.

Proof. Choose $g \in E(S)$ such that $e>g \geq f$ and $e$ covers $g$. Since $g S g \supseteq f S f$ it is enough to prove that $S$ can be embedded in the partial semigroup $e S e \backslash g S g$. 
Let $e=(A, 1)$. Then $g=(A \cup\{u a\}, 1)$ for some $u \in A$ and $a \in X \cup X^{-1}$, where the final letter in the reduced form of $u$ is not $a^{-1}$ and $u a \notin A$. Write

$$
r=\max \{l(w)+1: w \in A\},
$$

choose $b \in X \backslash\left\{a, a^{-1}\right\}$ (this is possible since $|X| \geq 2$ ) and define a subset $Y$ of $G$ (=FFG by

$$
Y=\left\{b^{-r} x b^{r}: x \in X \backslash\{b\}\right\} \cup\left\{a^{-r} b a^{r}\right\} .
$$

Every element of $Y$ has length $2 r+1$ (being reduced as written). Further, $Y \cap Y^{-1}=\varnothing$, since $X \cap X^{-1}=\varnothing$. Each element of $Y \cup Y^{-1}$ is fully determined by its initial segment of length $r+1$, and if $p, q \in Y \cup Y^{-1}$ are such that $p \neq q^{-1}$ then the cancellation in the product $p q$ does not reach the central factor of either $p$ or $q$. Thus the elements of $Y$ have central significant factors.

For all $y \in Y \cup Y^{-1}$, write

$$
B_{y}=A \cup \bar{y} \cup y A \text {. }
$$

Thus, for all $y \in Y \cup Y^{-1}$,

$$
\left(B_{y}, y\right)=(A, 1)(\bar{y}, y)(A, 1) \in e S e
$$

and

$$
\left(B_{y}, y\right)^{-1}=\left(B_{y^{-1}}, y^{-1}\right) .
$$

Let $K$ denote $\left\{\left(B_{y}, y\right): y \in Y\right\}$ and write $T=\left\langle K \cup K^{-1}\right\rangle$. Evidently $T \subseteq e S e$ and $|K|=|Y|=$ $|X|$. To establish the theorem it suffices to show that $K$ is a set of free generators of $T$ and that $T \cap \mathrm{gSg}=\varnothing$.

Since $Y \cap Y^{-1}=\varnothing$ it follows that $K \cap K^{-1}=\varnothing$; that is, $K$ satisfies condition (i) of Lemma 1. We prove next that $K$ also satisfies condition (ii). Let $v \in K \cup K^{-1}$ be such that

$$
\mathscr{S}(v) \subseteq \bigcup_{i=1}^{n} \mathscr{S}\left(v_{i, 1} v_{i, 2} \ldots v_{i, n_{i}}\right)
$$

where each $v_{i, j} \in K \cup K^{-1}$ and $v_{i, j}^{-1} \neq v_{i, j+1} \quad\left(j=1,2, \ldots, n_{i}-1 ; i=1,2, \ldots, n\right)$. Now $v \pi \in \mathscr{S}(v)$. Hence there exists $k \in\{1,2, \ldots, n\}$ such that

$$
v \pi \in \mathscr{S}\left(v_{k, 1} v_{k, 2} \ldots v_{k, n_{k}}\right) \text {. }
$$

For $j=1,2, \ldots, n_{k}$ let us write $y_{j}=v_{k, j} \pi$ and $B_{j}=B_{y_{j}}$ : thus

$$
v_{k, j}=\left(B_{j}, y_{j}\right) \quad\left(j=1,2, \ldots, n_{k}\right) \text {. }
$$

Hence

$$
v \pi \in B_{1} \cup y_{1} B_{2} \cup\left(y_{1} y_{2}\right) B_{3} \cup \ldots \cup\left(y_{1} y_{2} \ldots y_{n_{k}-1}\right) B_{n_{k}} .
$$

Suppose that $v \pi \notin B_{1}$. Then there exists $t \in\left\{1,2, \ldots, n_{k}-1\right\}$ such that $v \pi \in\left(y_{1} y_{2} \ldots y_{t}\right) B_{t+1}$ and so

$$
y_{t}^{-1} y_{t-1}^{-1} \ldots y_{1}^{-1}(v \pi) \in B_{t+1} \text {. }
$$


Now $y_{j}^{-1} \neq y_{j+1}(j=1,2, \ldots, t-1)$ (for otherwise $v_{k, j}^{-1}=v_{k, j+1}$ for some $j$, which is a contradiction); also $y_{1} \neq v \pi$, since $y_{1} \in B_{1}$ while $v \pi \notin B_{1}$. Hence the initial segment of $y_{t}^{-1} y_{t-1}^{-1} \ldots y_{1}^{-1}(v \pi)$ of length $r+1$ is just the initial segment of $y_{t}^{-1}$ of length $r+1$. Thus the initial segment of $y_{t}^{-1}$ of length $r+1$ lies in $B_{t+1}$, since $B_{t+1}$ is left closed.

Now $B_{t+1}=A \cup \bar{y}_{t+1} \cup y_{t+1} A$. Since no element of $A$ has length exceeding $r-1$, the only element of $B_{t+1}$ of length precisely $r+1$ is the initial segment of $y_{t+1}$ of this length. Hence $y_{t}^{-1}$ and $y_{t+1}$ have the same initial segment of length $r+1$ and so $y_{t}^{-1}=y_{t+1}$, which is a contradiction. Consequently $v \pi \in B_{1}$.

Since $v \pi \in Y \cup Y^{-1}$ and $B_{1}=A \cup \bar{y}_{1} \cup y_{1} A$, a similar argument to that above then shows that $v \pi=y_{1}$. Hence $v=\left(B_{1}, y_{1}\right)=v_{k, 1}$. We have thus proved that $K$ satisfies condition (ii) of Lemma 1 . Thus $T$ is free of rank $|K|$ and so $S \cong T$.

Finally, we show that $T \cap g S g=\varnothing$. Suppose that the conclusion is false and hence that there exists $(C, h) \in T \cap g S g$. As remarked earlier, $g=(D, 1)$, where $D=A \cup\{u a\}$. Since $(C, h) \in g S g$,

$$
(C, h)=\mathrm{g}(C, h) \mathrm{g}=(D \cup C \cup h D, h) .
$$

Thus $C=(D \cup h D) \cup C$ and so $D \cup h D \subseteq C$. In particular, $u a \in C$.

Since $(C, h) \in T$, there exist $y_{1}, y_{2}, \ldots, y_{n} \in Y \cup Y^{-1}$ (not to be confused with the $y_{i}$ in the first part of the proof) such that

$$
(C, h)=\left(B_{1}, y_{1}\right)\left(B_{2}, y_{2}\right) \ldots\left(B_{n}, y_{n}\right),
$$

where, for each $i$, we have written $B_{i}$ for $B_{y_{i}}$. But $u a \in C$. Hence

$$
u a \in B_{1} \cup y_{1} B_{2} \cup\left(y_{1} y_{2}\right) B_{3} \cup \ldots \cup\left(y_{1} y_{2} \ldots y_{n-1}\right) B_{n} .
$$

Suppose first that $u a \in B_{1}=A \cup \bar{y}_{1} \cup y_{1} A$. Since $u a \notin A$ we have that $u a \in \bar{y}_{1} \cup y_{1} A$. But since $l(u a) \leq r$, this implies that $u a$ is an initial segment of $y_{1}$ and hence is of the form $a^{-s}$ or $b^{-s}$ for some positive integer $s$. Thus $a=a^{-1}$ or $a=b^{-1}$ and in either case we have a contradiction.

Similarly, if $u a \in\left(y_{1} y_{2} \ldots y_{t}\right) B_{t+1}$ and $y_{1} y_{2} \ldots y_{t}=1$ for some $t \in\{1,2, \ldots, n-1\}$, we again obtain a contradiction. Thus, for some $t \in\{1,2, \ldots, n-1\}, u a \in\left(y_{1} y_{2} \ldots y_{t}\right) B_{t+1}$, where $y_{1} y_{2} \ldots y_{t} \neq 1$. Now we can write $y_{1} y_{2} \ldots y_{t}$ as a reduced word in $Y \cup Y^{-1}$ : thus

$$
y_{1} y_{2} \ldots y_{t}=z_{1} z_{2} \ldots z_{k}
$$

where each $z_{i} \in Y \cup Y^{-1}$ and $z_{i}^{-1} \neq z_{i+1}(i=1,2, \ldots, k-1)$. Hence

$$
u a \in z_{1} z_{2} \ldots z_{k} B_{t+1} .
$$

Let us write

$$
w=z_{k}^{-1} z_{k-1}^{-1} \ldots z_{1}^{-1} u a .
$$

Then $w \in B_{t+1}$. Now cancellation on the right-hand side of (1) cannot reach the central factor of any $z_{i}^{-1}$, since $z_{i}^{-1} \neq z_{i+1}(i=1,2, \ldots, k-1)$ and $l(u)<r$. Also it cannot reach the final letter $a$ since $l(u)<r$ and the final segment of $z_{1}^{-1}$ of length $r$ is either $a^{r}$ or $b^{r}$. 
Hence

$$
l(w) \geq r+3
$$

and the last letter of the reduced form of $w$ is $a$.

Since $w \in B_{t+1}=A \cup \bar{y}_{t+1} \cup y_{t+1} A$ and no word in $A$ has length exceeding $r-1$, (2) shows that

$$
w \in \bar{y}_{t+1} \cup y_{t+1} A \text {. }
$$

Hence the initial segments of $w$ and $y_{t+1}$ of length $r+2$ must coincide; also

$$
l(w) \leq(2 r+1)+(r-1)=3 r .
$$

Suppose that $k>1$. If there exist $i, j$ such that $z_{i}^{-1}=a^{-r} b a^{r}$ and $z_{j}^{-1}=b^{-r} x b^{r}$ for some $x \in\left(X \cup X^{-1}\right) \backslash\left\{b, b^{-1}\right\}$ then

$$
l(w) \geq(r+1)+2 r=3 r+1,
$$

which contradicts (4). Thus either

$$
z_{i}^{-1}=b^{-r} x_{i} b^{r} \quad \text { for some } \quad x_{i} \in\left(X \cup X^{-1}\right) \backslash\left\{b, b^{-1}\right\} \quad(i=1,2, \ldots, k)
$$

or

$$
z_{i}^{-1}=a^{-r} c a^{r} \quad(i=1,2, \ldots, k), \text { where } \quad c \in\left\{b, b^{-1}\right\} .
$$

In the first case

$$
w=b^{-r} x_{1} x_{2} \ldots x_{k} b^{r} u a,
$$

where $x_{i}^{-1} \neq x_{i+1}(i=1,2, \ldots, k-1)$, and so the initial segment of $w$ of length $r+2$ is $b^{-r} x_{1} x_{2}$. But the initial segments of $w$ and $y_{t+1}$ of length $r+2$ are the same. This gives a contradiction since $x_{2} \neq b$. In the second case, $w=a^{-r} c^{k} a^{r} u a$ and in the same way we again get a contradiction. Thus $k=1$.

It follows that the initial segments of $y_{t+1}$ and $z_{1}^{-1}$ of length $r+1$ coincide. Hence $y_{t+1}=z_{1}^{-1}$ and so

$$
w=y_{t+1} u a \text {. }
$$

Suppose that $w \in y_{t+1} A$. Then, from (5), $u a \in A$, which is false. Hence, from (3), $w \in \bar{y}_{t+1}$. Thus, since the last letter of $w$ (in reduced form) is $a$, we see from (2) that $w=a^{-r} c a^{s}$ and $y_{t+1}=a^{-r} c a^{r}$, where $c \in\left\{b, b^{-1}\right\}$ and $2 \leq s \leq r$. Hence, from (5),

$$
a^{-r} c a^{s}=a^{-r} c a^{r} u a
$$

and so $u=a^{-(r+1-s)}$. Since $s \leq r$ this contradicts the fact that the last letter in the reduced form of $u$ is not $a^{-1}$.

Consequently $T \cap g S g=\varnothing$ and the proof is complete.

Remark 1. The result fails for the case $|X|=1$ : for if $S=\mathscr{F} \mathscr{I}_{X}$, where $|X|=1$, and $e, f \in E(S)$ are such that $e$ covers $f$ then the set of idempotents in eSe $\backslash f S f$ is totally ordered, whereas $E(S)$ is not totally ordered. 
REMARK 2. Let $S$ be a free inverse semigroup of rank 1 and let $e \in E(S)$. Then $e S e \nsubseteq E(S)$. Choose a non-idempotent element $a \in e S e$. By [4, Corollary 2.5$],\left\langle\left\{a, a^{-1}\right\}\right\rangle$ is a free inverse subsemigroup of $S$ of rank 1 . This shows that $S$ can be embedded in $e S e$.

Remark 3. From Theorem 1 and Remark 2 we see that if $M$ is an ideal of a free inverse semigroup $S$ of arbitrary rank then $S$ can be embedded in $M$.

To conclude, we extend Theorem 1 with the aid of a result of O'Carroll's [3, p. 19], which we state below as Lemma 2. Recall that, by Schreier's theorem [1, Theorem 7.2.1], every subgroup of a free group is itself free.

LEMMA 2. Let $S=\mathscr{F} \mathscr{\Phi}_{X}$ and $G=\mathscr{F} \mathscr{G}_{X}$, where $X$ is a nonempty set. Let $H$ be a non-trivial subgroup of $G$. Then there exists a free inverse subsemigroup $T$ of $S$ such that rank of $T=$ rank of $H$.

Now let $c$ and $d$ be cardinal numbers such that $c \geq 2$ and $0<d \leq \max \left\{\kappa_{0}, c\right\}$. It can be shown that every free group of rank $c$ contains a (free) subgroup of rank $d$. Hence, by Lemma 2, every free inverse semigroup of rank $c$ contains a free inverse subsemigroup of rank $d$. From Theorem 1 we then immediately obtain

THEOREM 2. Let $S$ be a free inverse semigroup of rank $c$, where $c \geq 2$, and let $d$ be any cardinal number such that $0<d \leq \max \left\{\kappa_{0}, c\right\}$. Let $e, f \in E(S)$ be such that $e>f$. Then the partial semigroup eSe $\backslash f S f$ contains a free inverse subsemigroup of $S$ of rank $d$.

In particular, if $S$ is a free inverse semigroup of rank 2 and $e, f \in E(S)$ are such that $e>f$ then $e S e \backslash f S f$ contains a free inverse subsemigroup of $S$ of rank $\aleph_{0}$. This strengthens [4, Corollary 2.7].

Acknowledgement. This work was completed while I held a visiting appointment at Monash University. I am grateful to the university authorities and the Mathematics Department for providing financial support and excellent facilities for research.

\section{REFERENCES}

1. M. Hall, Jr. The theory of groups, (Macmillan, 1959).

2. J. M. Howie, An introduction to semigroup theory, (Academic Press, 1976). 17-23.

3. L. O'Carroll, A note on free inverse semigroups, Proc. Edinburgh Math. Soc. (2) 19 (1974),

4. N. R. Reilly, Free generators in free inverse semigroups, Bull. Austral. Math. Soc. 7 (1972), 407-424.

5. N. R. Reilly, Free generators in free inverse semigroups: Corrigenda, Bull. Austral. Math. Soc. 9 (1973), 479.

6. N. R. Reilly, Free inverse semigroups, Algebraic theory of semigroups, (Szeged, 1976), Colloq. Math. Soc. János Bolyai Vol. 20, 479-508.

7. H. E. Scheiblich, Free inverse semigroups, Semigroup Forum 4 (1972), 351-359.

8. H. E. Scheiblich, Free inverse semigroups, Proc. Amer. Math. Soc. 38 (1973), 1-7.

Mathematics Department

UNIVERSTTY OF GLASGOW

GLASGOW G12 8QW

SCOTLAND 